\title{
Functional Outcomes of Acute Inpatient Rehabilitation in Patients With Chronic Graft-Versus-Host Disease
}

\author{
Jason Leung, MD, Sean Smith, MD, Claire Kalpakjian, PhD, MS
}

\begin{abstract}
Background: Growing numbers of allogeneic stem cell transplants and improved posttransplant care have led to an increase of individuals with chronic graft-versus-host disease (cGVHD). Although cGVHD leads to functional impairment for many, there is limited literature regarding the benefits of acute inpatient rehabilitation for patients with CGVHD.

Objective: To assess Functional Independence Measure (FIM) outcomes of patients with CGVHD during acute inpatient rehabilitation and to compare inpatient rehabilitation outcomes with patients with burn injuries, a rehabilitation patient population with similar comorbidities.
\end{abstract}

Design: Retrospective chart review.

Setting: Acute rehabilitation center at a large academic medical center.

Patients (or Participants): A total of 37 adult patients with cGVHD and 30 with burn injuries admitted to inpatient rehabilitation from 2010 to 2015.

Methods or Interventions: Linear regression analysis to evaluate group (cGVHD versus burn) differences in functional gains. Effect size and minimal detectable change at the $90 \%$ confidence level (MDC90) were used to evaluate change in FIM outcomes.

Main Outcome Measurements: Total FIM gain, motor FIM gain, and FIM efficiency.

Results: Patients with CGVHD had statistically significant lower functional gains than patients with burn injuries, with an average of 11.66 fewer total FIM points $(P \leq .001), 10.54$ fewer motor FIM points $(P=.01)$, and 2.45 units less of FIM efficiency $(P=.01)$. At the time of discharge, $7(18 \%)$ patients with CGVHD exceeded the MDC90 values for total FIM gain versus 9 (30\%) patients with burn injuries $(P=.26)$. Eight $(21 \%)$ patients with CGVHD exceeded the MDC90 for motor FIM gain versus $13(43 \%)$ patients with burn injuries $(P=.048)$. Effect sizes for patients with CGVHD and with burn injury were moderate to large, respectively, with patients with burn injuries having nearly twice the magnitude of gains as patients with cGVHD.

Conclusions: Despite achieving more modest functional gains than patients with burn injuries, patients with cGVHD improved in function after acute inpatient rehabilitation. If replicated in larger studies, patients with functional impairment from cGVHD can be considered for inpatient rehabilitation. Future work should also determine minimal clinically important differences in function gain from inpatient rehabilitation for patients with cGVHD.

Level of Evidence: II

\section{Introduction}

Graft-versus-host disease (GVHD) is a common and potentially debilitating complication in patients who undergo allogeneic hematopoietic stem cell transplantation (HSCT) [1,2]. Used to treat hematologic malignancies and some autoimmune conditions, an allogeneic HSCT is when a host receives cells to reconstitute their immune system from a genetically similar donor. Despite attempts to match human leukocyte antigens between donor and host, the transferred immune cells may recognize the host's body as a foreign entity, thus creating the inflammatory GVHD process wherein the newly donated cells attack highly mitotic areas of the host. GVHD can be further classified as acute or chronic (ie, cGVHD), of which both are histologically distinct entities. Acute GVHD typically occurs within the first 100 days after a HSCT and mainly affects only the skin and gastrointestinal system. Conversely, symptoms of cGVHD are more pervasive and often more severe, potentially impacting multiple organ systems and causing profound functional impairment secondary to effects of the disease, including joint contracture, weakness, pain, and decreased cardiopulmonary function 
(Table 1). When cGVHD impacts fascial planes, fibrosis can occur, resulting in limiting range of motion, cramps, and pain. Unlike CGVHD, acute GVHD can be self-limiting and not necessarily progress to the chronic form. Furthermore, the treatment of cGVHD typically involves high-dose glucocorticoids as a first-line measure to reduce inflammation of the newly engrafted immune system. This treatment has the potential to cause steroid myopathy, decreased bone density, avascular necrosis, and raises the risk of infection, all of which have the potential to impair physical function [3].

The incidence of cGVHD is approximately $50 \%$ in patients undergoing allogeneic HSCT with an average onset of 7.4 months [4], and the number of patients with cGVHD continues to grow as the number of allogeneic HSCTs performed worldwide increases by $10 \%-20 \%$ annually $[5,6]$. Furthermore, as posttransplant care, including antirejection measures have improved, the number of older patients with chronic pretransplant comorbidities who undergo HSCT is increasing, and this frailer population is developing CGVHD. This underscores the need for effective rehabilitation interventions to reduce the impact on health and quality of life of persons undergoing HSCT, including multidisciplinary inpatient rehabilitation $[6,7]$. The exact benefit of comprehensive inpatient rehabilitation, however, is unclear in this population, and no studies have been published examining the outcomes of adult patients with $C G V H D$ receiving inpatient rehabilitation.

Comparing functional gains in inpatient rehabilitation of patients with CGVHD with a patient population who have similar impairments and pathophysiology that impact function, and for whom there is evidence of benefit from inpatient rehabilitation, can provide a frame of reference for exploring the degree of benefit from inpatient rehabilitation for patients with cGVHD. Among typical inpatient rehabilitation patients who may have similar symptoms impacting function are those who have sustained burn injuries. Like patients with cGVHD, patients with burn injuries also may develop skin fibrosis and joint contracture, are in a catabolic state causing muscle weakness through protein breakdown, have decreased cardiopulmonary performance, have impaired function, and are at risk for medical complications such as infection.

Emerging literature on the benefits of inpatient rehabilitation suggests that patients with burn injuries make functional gains [8] and benefit from earlier initiation of rehabilitation [9] and mobility training in acute care [10]. There is also some evidence that comorbidities and complications associated with burn injuries do not significantly mitigate functional gains during inpatient rehabilitation [11]. Other work suggests that patients with burn injuries feel that rehabilitation interventions have a long-term, positive impact on physical and psychosocial outcomes [12].

Although burn injuries are an acute, traumatic event and CGVHD is a chronic process, the rehabilitation approaches are similar and involve progressive mobility, aggressive range of motion, skin care, and family training. In this study, we examined whether change in function by discharge was comparable between patients with CGVHD and burn injuries and examined indices of clinical change to explore treatment effectiveness for these groups of patients.

\section{Methods}

A retrospective chart review of adult patients admitted to the University of Michigan acute inpatient rehabilitation unit directly from an acute medical service with a diagnosis of cGVHD or burn injury between 2010 and 2015 was performed. This study was approved by the University of Michigan Medical School institutional review board. Data collected included basic demographics (gender, age), length of stay during inpatient rehabilitation, and Functional Independence Measures (FIM) scores. The FIM scale is composed of the motor domain containing 13 items and cognitive domain containing 5 items with combined total scores ranging from 18 to 126. The FIM scale has been shown to have favorable psychometric properties across many impairment categories [13]. We included the length of stay and number of physical therapy (PT) and occupational

Table 1

Common rehabilitation issues in patients with CGVHD and with burn injuries

\begin{tabular}{lll}
\hline Organ System & Common Problems-cGVHD & Common Problems-Burn \\
\hline Skin/fascia & Sclerodermatous contractures, skin breakdown, rash & Contractures, skin breakdown, rash \\
Muscle & Steroid-induced myopathy, myositis & Weakness through increased catabolism \\
Bone/joint & Osteoporosis (risk of fractures), avascular necrosis & Heterotopic ossification \\
Gastrointestinal system & Malabsorptive diarrhea & Ileus \\
Peripheral nervous system & Peripheral neuropathy, mononeuropathy from fascial & Peripheral neuropathy, mononeuropathy from fascial \\
& thickening causing compression & thickening causing compression \\
Cardiopulmonary & Physical deconditioning, fatigue, obstructive lung & Physical deconditioning, fatigue, restrictive lung \\
& disease, left ventricular hypertrophy from & disease \\
Ocular & immunosuppressants & Thermal injury \\
Oral & Xerophthalmia & Thermal injury \\
\hline
\end{tabular}

cGVHD = chronic graft-versus-host disease. 
therapy sessions during acute medical care stay before acute rehabilitation admission to control for their effects. The nature of acute medical care varies for these 2 patient groups, with patients with burn injuries having far longer acute care stays and consequently receiving more therapy sessions.

\section{Statistical Analysis}

Descriptive terms were used to describe demographic characteristics and FIM admission and discharge scores by group (cGVHD versus burn). We used linear regression to examine whether there were significant differences in functional gains during inpatient rehabilitation (total FIM gain, motor FIM gain, and FIM efficiency) by group, controlling for other relevant factors, such as personal characteristics and health care services received before inpatient rehabilitation. For FIM efficiency, we tested a model of age, length of stay in acute care, and count of physical therapy and occupational therapy sessions during acute care and CGVHD patients compared to burn patients. For total FIM gain and motor FIM gain, we added length of stay in inpatient rehabilitation and admission total and motor FIM (respectively) to the model. Assumptions of linearity, independence of residuals, homoscedasticity, multicollinearity, outliers, and normality were checked for each regression analysis. In evaluating outliers, we examined cases with standardized residual $>3.0$, leverage values $>.20$, and/or Cook's distance $>1$. For FIM efficiency, the assumption of multicollinearity was violated with a tolerance value of .092 for the count of PT sessions, which was highly correlated with the count of occupational therapy sessions $(r=.942)$. Therefore, PT sessions were dropped from the regression model for all 3 functional outcomes. In addition, 2 cases (patients with burn injuries) were identified as possible outliers for FIM efficiency. We elected to retain these cases, as regression results did not substantially change when they were excluded. Assumptions for total FIM and motor FIM gain were all met and no outliers identified.

Because we also were interested in how much patients in each group would benefit from inpatient rehabilitation, we used effect size to quantify the magnitude of change during inpatient rehabilitation. We calculated within-group (cGVHD and burn) effect size as admission to discharge difference divided by the pooled standard deviation, a more conservative estimate of effect size. To determine the minimum amount of change in total and motor FIM scores that exceeded measurement error or the smallest change that is due to "true" change and not variation in measurement [14], we calculated the minimal detectable change at the $90 \%$ confidence level (MDC90). We used the $\chi^{2}$ test of homogeneity to test group differences in the percentage of patients whose total and motor FIM gain scores exceeded the MDC90. All analyses were conducted in SPSS, version 23 (IBM Corp, Armonk, NY).

\section{Results}

\section{Patient Characteristics}

Of 72 patients, 67 patients were included in this analysis, 37 with CGVHD and 30 with burn injuries. Patients transferred off of service due to complications before being able to fully assess FIM scores were not included in the analysis. There were 5 patients with cGVHD who were excluded because of transfer before discharge due to complications (ie, fluid overload, gastrointestinal bleeding, severe upper gastrointestinal symptoms, and altered mental status). No patients with burn injuries were unexpectedly transferred (Table 2 ).

\section{Functional Gains in Inpatient Rehabilitation}

For all FIM outcomes, group (cGVHD versus burn) was statically significant such that patients with CGVHD made fewer gains in FIM efficiency, total FIM, and motor FIM compared with patients with burn injuries. On average, patients with CGVHD had 2.47 units lower FIM efficiency, 11.66 fewer total FIM gain points, and 10.54 fewer motor FIM gain points; see Table 3 .

\section{Magnitude of Change and Minimal Detectable Change During Inpatient Rehabilitation}

Effect sizes for cGVHD and patients with burn injuries were moderate and large, respectively, with the patients with burn injury having nearly twice the magnitude of functional gain as the patients with CGVHD [15]. The MDC90 values for total and motor FIM scores were slightly greater for patients with burns (8.5 points for total FIM

Table 2

Patient characteristics

\begin{tabular}{lcc}
\hline Characteristic & CGVHD $(\mathrm{n}=37)$ & Burn $(\mathrm{n}=30)$ \\
\hline Age & $56.25(13.4), 21-77$ & $43.03(16.6), 17-76$ \\
Gender, male $(\mathrm{n}, \%)$ & $20(54.1)$ & $23(76.7)$ \\
$\begin{array}{l}\text { Length of stay in } \\
\text { inpatient }\end{array}$ & $13.68(11.8)$ & $14.57(11.6)$ \\
$\quad$ rehabilitation, d & & \\
Length of stay in & $33.17(29.2), 4-115$ & $55.73(49.0), 10-227$ \\
$\quad$ acute medical care, d & & \\
Admit total FIM & $67.19(18.3), 20-96$ & $67.27(18.2), 22-98$ \\
Discharge total FIM & $80.73(23.1), 23-111$ & $92.03(20.2), 27-113$ \\
Admit motor FIM & $40.35(13.6), 14-66$ & $39.4(13.3), 13-69$ \\
Discharge motor FIM & $52.46(17.9), 16-76$ & $61.67(15.7), 20-79$ \\
FIM efficiency & $1.22(1.1), 0-5$ & $2.71(2.0), 0-8$ \\
Count PT sessions in & $10.08(10.8), 0-34$ & $40.93(20.4), 8-84$ \\
$\quad$ acute care & & \\
Count OT sessions in & $7.16(9.7), 0-50$ & $42.03(21.7), 12-90$ \\
$\quad$ acute care & & \\
\hline
\end{tabular}

Values are reported as mean, standard deviation, and range unless otherwise noted.

cGVHD = chronic graft-versus-host disease; FIM = Functional Independence Measure; PT = physical therapy; OT = occupational therapy. 
Table 3

Regression analyses for FIM efficiency, FIM total gain, and FIM motor gain

\begin{tabular}{lcccc}
\hline & Coeff. & $P$ Value & $95 \mathrm{CI}$ LB & $95 \mathrm{Cl}$ UB \\
\hline FIM efficiency* & & & & \\
$\quad$ Group (cGVHD vs burn) & -2.48 & $<.001$ & -3.64 & -1.31 \\
FIM total score & \\
$\quad \begin{array}{l}\text { Group (cGVHD vs burn) } \\
\quad\end{array}$ & -11.67 & .01 & -20.86 & -2.47 \\
$\begin{array}{l}\text { Motor FIM score } \\
\quad \text { Group (cGVHD vs burn) }\end{array}$ & -10.55 & .01 & -18.73 & -2.37 \\
\hline
\end{tabular}

FIM = Functional Independence Measure; Coeff = coefficient; $95 \mathrm{CI}$ LB $=$ lower bound of the $95 \%$ confidence level; $95 \mathrm{CI} \mathrm{UB}=$ upper bound of the $95 \%$ confidence level; cGVHD = chronic graft-versus-host disease; OT = occupational therapy.

* Adjusted for age, length of stay (days) in acute care, and count of OT sessions in acute care.

$\dagger$ Adjusted for age, length of stay (days) in acute care, admit total FIM, and count of OT sessions in acute care; length of stay (days) on inpatient rehabilitation was statistically significant for FIM Total score (coeff. $=0.49, P<.001$ ) and Motor FIM score (coeff. $=0.42, P<.001$ ).

and 4.5 points for motor FIM). At the time of discharge, 7 $(18.9 \%)$ patients with CGVHD exceeded the MDC90 values for total FIM gain compared with $8(26.7 \%)$ patients with burn injuries, which was not statistically significant $\left(\chi^{2}=\right.$ 1.24, $P=.26)$. Eight $(21.6 \%)$ patients with cGVHD exceeded the MDC 90 for motor FIM gain compared with 13 (43.3\%) patients with burn injuries, which was statistically significant $\left(\chi^{2}=3.89, P=.048\right.$; see Table 4$)$.

\section{Discussion}

Patients with CGVHD in this study did not make as much functional gain as those with burn injuries. When we accounted for therapy services provided while patients received medical care before entering rehabilitation, the effect sizes of motor and total FIM gain for these patients with cGVHD suggest that, on average, they do benefit from inpatient rehabilitation to improve function. Although patients with CGVHD have similar comorbidities to those with burn injuries, such as skin contractures and cardiopulmonary limitations [3], CGVHD is a chronic, progressive illness that, along with glucocorticoid treatment and medical complications such as infection, can compromise function over a longer period of time.

In contrast, burn injuries typically occur in otherwise-healthy individuals and is not a progressive process. This may account, in part, for the greater gains made by patients with burn injuries during inpatient rehabilitation, even when, on average, both groups of patients had similar levels of function at the time of admission. Moreover, patients with more severe cGVHD may have less functional gain with acute inpatient rehabilitation than those with mild disease, which we did not evaluate in this study; future studies should evaluate the severity of disease vis-à-vis inpatient rehabilitation gains. Of course, patients requiring acute inpatient rehabilitation have significant functional impairment necessitating hospitalization, so patients with more mild disease may not require intensive medical rehabilitation. Given that the benefits of inpatient rehabilitation in patients who have sustained major burn injuries is well-documented, it is encouraging that in this preliminary investigation, patients with CGVHD made gains, albeit to a lesser degree, when beginning at a similar level of function as patients with burn injuries. If similar gains in patients with cGVHD are replicated in larger studies, providers can consider this population as one that benefits from inpatient rehabilitation.

Although our preliminary data suggest that patients with CGVHD do benefit from inpatient rehabilitation, more work is needed to determine thresholds of minimal clinically important differences (MCIDs). In this study, we only examined whether gains exceeded measurement error, not the degree to which changes exceeded a meaningful threshold. This is an important area for development, given the expected growth of patients who have undergone HSCT, many of whom will go on to develop cGVHD. Distribution-based and anchorbased methods to determine MCIDs [16] and external responsiveness indices [15] for the FIM in patients with cGVHD and other measures of function during inpatient rehabilitation are a critical step to evaluate the

Table 4

Admission and discharge scores, ICC, SEM, effect size, and MDC90 for total and motor FIM gain by cGVHD and burn injury groups

\begin{tabular}{|c|c|c|c|c|c|}
\hline & Admission Mean (SD) & Discharge Mean (SD) & ICC $(95 \% \mathrm{Cl})$ & Effect Size $^{*}(95 \% \mathrm{Cl})$ & $M D C 90^{-}$ \\
\hline \multicolumn{6}{|l|}{ Total FIM } \\
\hline CGVHD & $67.19(18.3)$ & $80.72(23.1)$ & $0.69(0.02,0.89)^{\ddagger}$ & $0.60(0.41,0.79)^{\ddagger}$ & 23.34 \\
\hline Burn & $67.27(18.2)$ & $92.03(20.2)$ & $0.43(-0.08,0.78)^{\ddagger}$ & $1.627(.951 .07,1.592 .24)^{\ddagger}$ & 31.84 \\
\hline \multicolumn{6}{|l|}{ Motor FIM } \\
\hline CGVHD & $40.35(13.6)$ & $52.45(17.9)$ & $0.59(-0.01,0.83)^{\ddagger}$ & $0.71(0.47,0.96)^{\ddagger}$ & 20.30 \\
\hline Burn & $39.40(13.3)$ & $61.67(15.7)$ & $0.36(-0.07,0.72)^{\ddagger}$ & $1.4936(1.13 .78,1.869)^{\ddagger}$ & 24.86 \\
\hline
\end{tabular}

$\mathrm{ICC}=$ intraclass correlation; SEM = standard error of the mean; MDC $90=$ minimal detectable change at the $90 \%$ confidence level; FIM = Functional Independence Measure; $\mathrm{CGVHD}=$ chronic graft-versus host disease; SD = standard deviation; $\mathrm{Cl}=$ confidence interval.

* Standardized effect size; calculated as discharge - admission score / pooled SD.

$\dagger$ Calculated as $1.65^{*} \mathrm{SEM}^{*} \sqrt{2}$.

$\neq P \leq .001$. 
effectiveness of rehabilitation interventions for these patients.

Finally, despite the potentially profound functional impairment associated with CGVHD, this diagnosis is not currently included in the Centers for Medicare and Medicaid Service (CMS) guidelines mandating that $60 \%$ of all patients on an acute inpatient rehabilitation unit have 1 of 13 specific diagnoses that necessitate acute inpatient rehabilitation [17]. Because patients with cGVHD do not qualify in the CMS rule, it is possible that these patients are being undertreated and receiving their rehabilitation care in suboptimal or less-intensive rehabilitation facilities. Because our data suggest that patients with cGVHD do show improvement in function with acute inpatient rehabilitation, and if these results are replicated in larger studies, these patients should be also prioritized as one of the standard CMS acute inpatient rehabilitation diagnoses.

Although our preliminary results are promising, there are several limitations to this study that should be taken into account. The sample size is small and is not representative of either population, as data were drawn from a single tertiary care academic hospital. An inherent limitation in the change indices we used is that changes in the measure (FIM) may not necessarily correspond to changes in an external clinical or health status measure at the individual patient level. Rather, they examine the extent of change over 2 occasions (admission and discharge) and may not reflect meaningful change in the condition of the patient [15]. Future work to develop MCIDs for the FIM and other measures of function is an important area for development.

\section{Acknowledgments}

The authors thank Steve Schilling, PhD, for his assistance with statistical analysis.

\section{References}

1. Lee SJ, Vogelsang G, Flowers ME. Chronic graft-versus-host disease. Biol Blood Marrow Transplant 2003;9:215-233.

2. Higman MA, Vogelsang GB. Chronic graft versus host disease. $\mathrm{Br} \mathrm{J}$ Haematol 2004; 125:435-454.

3. Smith SR, Haig AJ, Couriel DR. Musculoskeletal, neurologic, and cardiopulmonary aspects of physical rehabilitation in patients with chronic graft-versus-host disease. Biol Blood Marrow Transplant 2015;21:799-808.

4. Arora M, Cutler CS, Jagasia MH, et al. Late acute and chronic graftversus-host disease after allogeneic hematopoietic cell transplantation. Biol Blood Marrow Transplant 2016;22:449-455.

5. Pasquini MC, Aljurf MD, Confer DL, et al. Global hematopoietic stem cell transplantation (HSCT) at one million: An achievement of pioneers and foreseeable challenges for the next decade. A report from the Worldwide Network For Blood and Marrow Transplantation (WBMT). Blood 2013;122:2133-2133.

6. Arai S, Arora M, Wang T, et al. Increasing incidence of chronic graft-versus-host disease in allogeneic transplantation: A report from the Center for International Blood and Marrow Transplant Research. Biol Blood Marrow Transplant 2015;21:266-274.

7. Wolff D, Bertz H, Greinix H, Lawitschka A, Halter J, Holler E. The treatment of chronic graft-versus-host disease: Consensus recommendations of experts from Germany, Austria, and Switzerland. Dtsch Arztebl Int 2011;108:732-740.

8. Sliwa JA, Heinemann A, Semik P. Inpatient rehabilitation following burn injury: Patient demographics and functional outcomes. Arch Phys Med Rehabil 2005;86:1920-1923.

9. Gomez M, Tushinski M, Jeschke MG. Impact of early inpatient rehabilitation on adult burn survivors' functional outcomes and resource utilization. J Burn Care Res 2017;38:E311-E317.

10. Deng $\mathrm{H}$, Chen J, Li F, et al. Effects of mobility training on severe burn patients in the BICU: A retrospective cohort study. Burns 2016;42:1404-1412.

11. Schneider JC, Gerrard P, Goldstein R, et al. The impact of comorbidities and complications on burn injury inpatient rehabilitation outcomes. PM R 2013;5:114-121.

12. Yohannan SK, Ronda-Velez Y, Henriquez DA, et al. Burn survivors' perceptions of rehabilitation. Burns 2012;38:1151-1156.

13. Stineman MG, Shea JA, Jette A, et al. The Functional Independence Measure: Tests of scaling assumptions, structure, and reliability across 20 diverse impairment categories. Arch Phys Med Rehabil 1996;77:1101-1108.

14. Copay AG, Subach BR, Glassman SD, Polly DW Jr, Schuler TC. Understanding the minimum clinically important difference: A review of concepts and methods. Spine J 2007;7:541-546.

15. Husted JA, Cook RJ, Farewell VT, Gladman DD. Methods for assessing responsiveness: A critical review and recommendations. J Clin Epidemiol 2000;53:459-468.

16. Mallinson T, Pape TL, Guernon A. Responsiveness, minimal detectable change, and minimally clinically important differences for the Disorders of Consciousness Scale. J Head Trauma Rehabil 2016;31:E43-E51.

17. Centers for Medicare \& Medicaid Services. Inpatient Rehabilitation Facility Prospective Payment System. Washington, DC: U.S. Department of Health and Human Services; 2017.

This journal-based CME activity is designated for 1.0 AMA PRA Category 1 Credit $^{\mathrm{TM}}$ and can be completed online at wWW.me. aapmr.org. This activity is FREE to AAPM\&R members and available to nonmembers for a nominal fee. For assistance with claiming CME for this activity, please contact (847) 737-6000.

\section{Disclosure}

J.L. Department of Physical Medicine and Rehabilitation, University of Michigan Medical School, Ann Arbor, MI

Disclosure: nothing to disclose

S.S. Department of Physical Medicine and Rehabilitation, University of Michigan Medical School, 325 E. Eisenhower Parkway, Suite 200, Ann Arbor, MI 48108. Address correspondence to: S.S.; e-mail: srsz@umich.edu

Disclosure: nothing to disclose
C.K. Department of Physical Medicine and Rehabilitation, University of Michigan Medical School, Ann Arbor, MI Disclosure: nothing to disclose

Funding to partially support this study was received from the University of Michigan Health System-Ann Arbor Center for Independent Living Advanced Rehabilitation Research Training Program, U.S. Department of Education, National Institute of Disability and Rehabilitation Research (H133P090008). 
This study was presented, in part, at the American Academy of Physical Medicine and Rehabilitation Annual Assembly.
Peer reviewers and all others who control content have no financial relationships to disclose.

Submitted for publication May 26, 2017; accepted November 2, 2017.

\section{CME Question}

Which of the following features is common to chronic graft versus host disease (cGVHD)?
a. Joint contractures.
b. Heterotopic ossification.
c. Small bowel obstruction.
d. Restrictive lung disease.

Answer online at me.aapmr.org 